\title{
Optimized Synthesis of Novel Pyrazole Based Thiazole Derivatives and their Antimicrobial Evaluation
}

\author{
N C Desai* and Malay J Bhatt \\ Division of Catalysis for Medicinal Chemistry, \\ Department of Chemistry (DST-FIST Sponsored \& UGC NON-SAP), \\ Mahatma Gandhi Campus, Maharaja Krishnakumarsinhji Bhavnagar University, \\ Bhavnagar-364002, India \\ E-mail: dnisheeth@rediffmail.com \\ Tel. No. 0278-2439852
}

Keywords: Pyrazole, thiazolidinone, process optimization, MIC, antimicrobial activity.

\begin{abstract}
A series of nitro pyrazole based thiazole derivatives compounds were synthesized by using solid base catalyst. The process was further optimized by setting up the solvent and catalyst ratio. The structures of these compounds were elucidated by spectral (IR, ${ }^{1} \mathrm{H} N M R,{ }^{13} \mathrm{C}$ NMR, mass spectra) analysis. Synthesized compounds were screened for their in vitro antibacterial activity against the representative panel of Gram-positive (Staphylococcus aureus, Streptococcus pyogenes) and Gram-negative (Escherichia coli, Pseudomonas aeruginosa) bacteria. These compounds were tested for their inhibitory action against strains of fungi (Candida albicans, Aspergillus niger, Aspergillus clavatus).
\end{abstract}

\section{Introduction}

As rate of microbial infections is increasing and microorganisms are resisting the known antimicrobial agents, designing novel, potent and broad spectrum antimicrobial agents are still remained a major challenge for medicinal chemists.

Pyrazoles belong to the family of five-member azoles, Pyrazole ring has attracted much attention as it has become fairly accessible and showed diverse properties. Pyrazoles and its derivatives are well known for their biological and medicinal, applications, such as antimicrobial [1], antitumor [2], antileukemia [3], antidepressant [4], anticonvulsant

[5], antifungal [6] and antitubercular [7]. Pyrazole analogoues can selectively inhibit (cyclooxygenase enzyme) COX-2 [8] they are also expressing anti-inflammatory, analgesic, antihypertensive, antipyretic, sedatives, and antidiabetic activities [9,10].

Pyrazolines are dihydro-pyrazole derivatives. They are also found to be equally pharmacologically interesting characteristic. Pyrazolines displayed a broad spectrum of potential pharmacological activities and are present in a number of pharmacologically active molecules. Some of major activities are listed as anti-inflammatory [11], anti-parasitary [12], anticonvulsant [13], antimicrobial [14], antinociceptives [15], antimalarial [16], antiviral [17], antioxidant [18], antiamoebic [19], cytotoxic [20], antidiabetic [21], antifungal [22], antinociceptive [23], antimycobacterial [24], antihepatotoxic [25] and pesticidal properties [26]. Edaravone is a neuroprotective agent and used for brain ischemia. Phenazone is an analgesic and antipyretic drug.

Thiazole derivatives are known to exhibit diverse bioactivities [27-30] such as antibiotic, anthelmintic, fungicidal, analgesic, anti-inflammatory, psychotropic, antioxidant, selective adenosine receptor antagonists, antipyretic etc. 4-Thiazolidones are undergoing different stages of clinical trials as potential thyromimetic, [31] antimicrobial, anti-HIV, anti-ischaemic, cardiovascular, anticancer, thrombolytic drugs. 


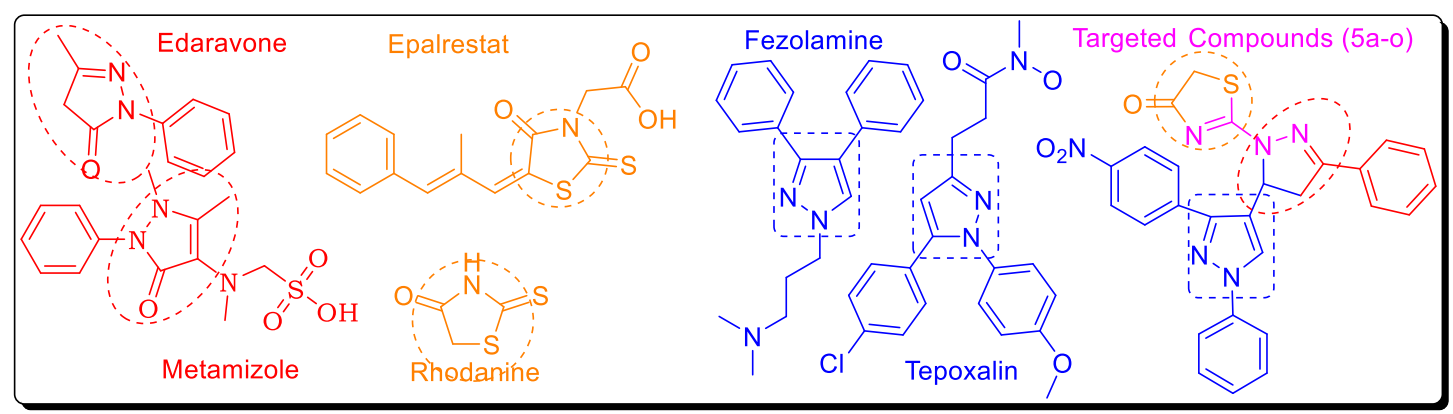

Figure 1 Structural resemblance between marketed drugs and targeted compounds

\section{Results and discussion}

\subsection{Chemistry}

To compare the efficienty of the catalyst, we had carried out a set of experiments by varying the reaction time, amounts of the catalysts and various solvents. The optimized conditions to prepare compound (3a-o) was achieved with $20 \mathrm{mmol}$ catalyst and dry methanol as solvent. The details are given below in Table 1 and Table 2. The final compounds (5a-o) can be synthesized in three main reaction steps. According to Scheme 1, the key chalcone derivatives (3a-0) are used as precursors for the synthesis of title compounds (5a-o). These chalcone derivatives were prepared from 1 (3-(4-nitrophenyl)-1-phenyl-1 $H$-pyrazole-4-carbaldehyde) and derivatives of acetophenone (2a-o). This reaction is modified Claisen Schmidt reaction which was further optimized. In second step, these chalcones (3a-o) were cyclise with thiosemicarbazide to get pyrazoline compounds (4ao). In the last step, compounds (4a-o) were further cyclised using ethyl bromoacetate in order to get targeted compounds (5a-o).

\subsection{Spectral characterization of synthesized compound (5a-0)}

IR spectrum of the compound 5a showed stretching vibrations at 3068, $3042 \mathrm{~cm}^{-1}$ due to the $\mathrm{C}-\mathrm{H}$ stretching of aromatic ring. Aromatic carbon having $\mathrm{C}-\mathrm{H}$ stretching of methylene group appeared at $2860 \mathrm{~cm}^{-1}>\mathrm{C}=\mathrm{O}$ stretching of thiazolidinone ring appeared at $1690 \mathrm{~cm}^{-1}$. Stretching vibration at $1578 \mathrm{~cm}^{-1}$ revealed the presence of $>\mathrm{C}=\mathrm{N}$ - group in the compound $5 \mathrm{a} .>\mathrm{C}=\mathrm{C}$ - stretching of aromatic ring appeared at $1532 \mathrm{~cm}^{-1}$. Asymmetric and symmetric stretching appeared at $1485 \mathrm{~cm}^{-}$ ${ }^{1}$ and $1351 \mathrm{~cm}^{-1}$ is due to the $-\mathrm{NO}_{2}$ group.

${ }^{1} \mathrm{H}$ NMR spectra of compound 5a showed a singlet at $\delta=8.32 \mathrm{ppm}$ which is the proof of $=\mathrm{C} \underline{\mathrm{H}}-\mathrm{N}<$ proton in pyrazoline ring. Aromatic proton expressed multiplet values between $\delta=6.86-$ $8.21 \mathrm{ppm}$. Functional group $=\mathrm{CH}-\mathrm{N}<$ of pyrazoline ring appeared as doublet of doublet at $\delta=6.09$ ppm. Protons of $-\mathrm{S}-\mathrm{CH}_{2}$ - gave a sharp singlet at $\delta=4.11 \mathrm{ppm}$. Lastly- $\underline{\mathrm{C}}_{2}-$ protons of pyrazoline ring appeared as two different doublet of doublet signals at $\delta=3.91 \mathrm{ppm}$ and at $\delta=3.42 \mathrm{ppm}$.

${ }^{13} \mathrm{C}$ NMR spectra of compound 5a showed distinct values of differently functionalized carbon atom. At $\delta=187.4 \mathrm{ppm}$ shows the presence of ketonic carbon in thiazolidinone ring $\left(-\mathrm{CH}_{2}-\right.$

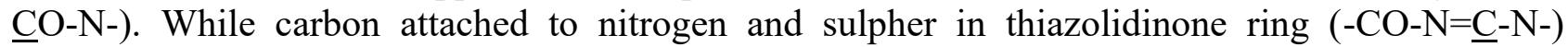
appeared at $\delta=178.0 \mathrm{ppm}$. Carbon attached to the phenyl ring in pyrazoline $(-\mathrm{N}=\underline{\mathrm{C}}-\mathrm{Ph})$ reveals its presence at $\delta=152.6 \mathrm{ppm}$. Carbon of 4-nitrophenyl ring attached to nitro group (Ar- $\left.\underline{\mathrm{C}}-\mathrm{NO}_{2}\right)$ gave signal at $\delta=148.2 \mathrm{ppm}$. Different aromatic carbons had given chemical shifts between $\delta=115.4$ $151.3 \mathrm{ppm}$. Chiral carbon of pyrazoline ring $\left(-\mathrm{N}-\underline{\mathrm{CH}}-\mathrm{CH}_{2^{-}}\right)$is responsible for chemical shift at $\delta=$ $60.4 \mathrm{ppm}$. Methylene carbon of pyrazoline ring $\left(-\mathrm{N}-\mathrm{CH}-\mathrm{CH}_{2}-\mathrm{CH}-\mathrm{Ph}\right)$ had given chemical shift at $\delta$ $=42.3 \mathrm{ppm}$. Methylene carbon of thiazolidinone ring $\left(-\mathrm{S}-\underline{\mathrm{C}} \mathrm{H}_{2}-\mathrm{CO}-\mathrm{N}-\right)$ gave shift at $\delta=39.0 \mathrm{ppm}$. 
Table 1 Effect of solvent on synthesis of compound $\mathbf{3} \mathbf{a}^{\mathrm{a}}$

\begin{tabular}{clcc}
\hline Entry & Solvent & Time $^{\mathrm{b}}(\min )$ & Yield $^{\mathrm{c}}(\%)$ \\
\hline 1 & Acetonitrile & 360 & 47 \\
2 & Ethanol (96\%) & 300 & 54 \\
3 & DMF & 280 & 40 \\
4 & THF & 250 & 35 \\
5 & DMSO & 300 & 61 \\
6 & Methanol & 280 & 67 \\
7 & Methanol Dry & 360 & 70 \\
\hline
\end{tabular}

a. Indole-3-carbaldehyde $(0.75 \mathrm{~mol})$, ethyl acetoacetate $(0.75 \mathrm{~mol})$ and thiourea $(0.5 \mathrm{~mol})$

b. All reactions were refluxed in ethanol till completion as indicated by TLC.

c. Isolated yield.

Table 2 Optimization of catalysts ${ }^{\mathrm{a}}$

\begin{tabular}{clccc}
\hline Entry & Catalyst & $\begin{array}{c}\text { Amount of catalyst } \\
\text { (mmol) }\end{array}$ & $\begin{array}{c}\text { Time }^{\mathbf{b}} \\
(\mathbf{m i n})\end{array}$ & $\begin{array}{c}\text { Isolated yield } \\
\text { (\%) }\end{array}$ \\
\hline 1 & $\mathrm{NaOH}$ & 20 & 480 & 89 \\
2 & $\mathrm{KOH}$ & 25 & 360 & 88 \\
3 & $\mathrm{Cs}_{2} \mathrm{CO}_{3}$ & 20 & 480 & 94 \\
4 & $\mathrm{Cs}_{2} \mathrm{CO}_{3}$ Recycled & 18 & 480 & 90 \\
5 & $\mathrm{CaO}$ & 20 & 360 & 52 \\
6 & $\mathrm{ZnO}$ & 20 & 600 & 55 \\
7 & $\mathrm{Hydrotelsite}$ & 25 & 900 & 10 \\
& $\mathrm{Mg}_{6} \mathrm{Al}_{2}\left(\mathrm{CO}_{3}\right)(\mathrm{OH})_{16} 4 \mathrm{H}_{2} \mathrm{O}$ & & & \\
8 & $\mathrm{RbOH}_{9}$ & 15 & 600 & 87 \\
10 & $\mathrm{Pd}_{2}(\mathrm{OAc})_{2}$ & 20 & 480 & 70 \\
$\mathrm{~K}_{2} \mathrm{CO}_{3}$ & 15 & 360 & 35 \\
\hline
\end{tabular}

a. 3-(4-nitrophenyl)-1-phenyl-1 $H$-pyrazole-4-carbaldehyde $(0.75 \mathrm{~mol})$ and acetophenone $(0.75 \mathrm{~mol})$.

b. All reactions were refluxed in ethanol till completion as indicated by TLC.<smiles>O=Cc1cn(-c2ccccc2)nc1-c1ccc([N+](=O)[O-])cc1</smiles>

(1)<smiles>[R]c1cccc(C(C)=O)c1</smiles>

$(2 a-0)$<smiles>[R]c1cccc(C(=O)/C=C/c2cn(-c3ccccc3)nc2-c2ccc([N+](=O)[O-])cc2)c1</smiles>

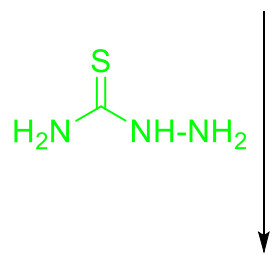

$0.1 \mathrm{~N} \mathrm{NaOH}$ Ethanol

Reflux $8 \mathrm{~h}$<smiles>[R]c1ccc(C2=NN(C3=NC(=O)CS3)C(c3cn(-c4ccccc4)nc3-c3ccc([N+](=O)[O-])cc3)C2)cc1</smiles>

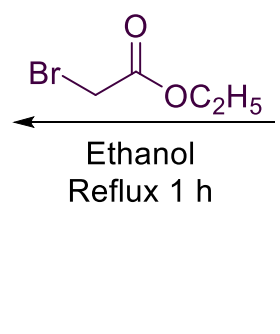<smiles>[R]c1ccc(C2=NN(C(N)=S)C(c3cn(-c4ccccc4)nc3-c3ccc([N+](=O)[O-])cc3)C2)cc1</smiles>

Scheme 1 Synthetic route for preparation of title compounds (5a-o) 


\subsection{Antibacterial activity}

From the Table 3 it has been clearly seen that synthesized compounds (5a-o) showed significant activity against bacterial strains along with MIC values of reference compounds ciprofloxacin. Compound containing electron donating groups at para position (compound $\mathbf{5 j}, \mathbf{5 m}$ and $\mathbf{5 0}$ ) had shown optimum activity against the bacterial strains. When para position is replaced by electron withdrawing group (compound $\mathbf{5 e}$ and $\mathbf{5 h}$ ) the activity was slightly decreased.

\subsection{Antifungal activity}

In comparison of the antifungal activity the case was altered. Compound containing electron withdrawing group at para position (compound 5e) had expressed excellent overall activity as compared to the standard drug nystatin. While compound $\mathbf{5 m}$ containing electron donating group at para position found to be a bit less active then compound 5e.

The remaining compounds of the series possessed feeble antimicrobial activity.

Table 3 Antimicrobial evaluation of synthesized compounds*

\begin{tabular}{clccccccc}
\hline & \multicolumn{1}{c}{ Minimal bactericidal concentration $(\boldsymbol{\mu g} / \mathbf{m L})$} & & \multicolumn{3}{c}{ Minimal fungicidal concentration } \\
$(\boldsymbol{\mu} \mathbf{g} / \mathbf{m L})$
\end{tabular}

* The protocol for the bio-activity measurement is as per literature method. [32-33]

E.c.- Escherichia coli MTCC 443; P.a.- Pseudomonas aeruginosa MTCC 1688, S.a.- Staphylococcus aureus MTCC 96; S.p.- Staphylococcus pyogenes MTCC 442; C.a.- Candida albicans MTCC 227; A.n.- Aspergillus niger MTCC 282; A.c.- Aspergillus clavatus MTCC 1323.

\section{Experimental}

\subsection{Materials and method}

The essential chemicals were purchased from E. Merck. Melting points were recorded on Gallenkamp apparatus and were left uncorrected. The completion of reaction was checked on aluminum-coated TLC plates $60, \mathrm{~F}_{245}$ (E. Merck) using various solvent systems as mobile phase and visualized under ultraviolet (UV) light, or iodine vapor. Elemental analysis $(\% \mathrm{C}, \mathrm{H}, \mathrm{N})$ was carried out by a Perkin-Elmer $2400 \mathrm{CHN}$ analyzer. IR spectra were also recorded on Perkin Elmer FT-IR L1280002 spectrophotometer. ${ }^{1} \mathrm{H}$ NMR and spectra were recorded on Varian Gemini 400 $\mathrm{MHz}$ and ${ }^{13} \mathrm{C}$ NMR spectra on Varian Mercury-400, $100 \mathrm{MHz}$ in DMSO- $d_{6}$ as a solvent and tetramethylsilane (TMS) as an internal standard. Mass spectra were scanned on a Shimadzu LCMS 2010 spectrometer. 


\subsection{Synthesis of 3-(4-nitrophenyl)-1-phenyl-1H-pyrazole-4-carbaldehyde (1)}

3-(4-nitrophenyl)-1-phenyl-1H-pyrazole-4-carbaldehyde (1) was prepared according to the literature method [34].

\subsection{Synthesis of 3-(3-(4-nitrophenyl)-1-phenyl-1H-pyrazol-4-yl)-1-arylprop-2-en-1-ones (3a-o)}

A mixture of 3-(4-nitrophenyl)-1-phenyl- $1 H$-pyrazole-4-carbaldehyde 1 (0.01 mol) and different derivative of acetophenone (2a-o) $(0.01 \mathrm{~mol})$ were stirred in presence of $\mathrm{CsCO}_{3} 20 \mathrm{~mol} \%$ as a catalyst using $45 \mathrm{~mL}$ dry methanol for $6 \mathrm{~h}$ at $55-60{ }^{\circ} \mathrm{C}$ on a heating pan with magnetic stirrer (Complete optimization is shown in Table 1 and 2). Product was filtered off, washed with water and crystallized from ethanol (95\%).

3.4. Synthesis of 3'-(4-nitrophenyl)-1'-phenyl-5-(aryl)-3,4-dihydro-1'H,2H-[3,4'-bipyrazole]-2carbothioamides (4a-o)

3'-(4-nitrophenyl)-1'-phenyl-5-(aryl)-3,4-dihydro-1'H,2H-[3,4'-bipyrazole]-2- carbothioamide (4a-o) were prepared according to the literature method [34].

3.5. General synthesis of 2-(3'-(4-nitrophenyl)-1'-phenyl-5-(aryl)-3,4-dihydro-1'H,2H-[3,4'bipyrazol]-2-yl)thiazol-4(5H)-ones (5a-o)

To suspension of compound (4a-o) $(0.01 \mathrm{~mol})$ in ethanol $(95 \%)$, ethyl bromoacetate $(0.01 \mathrm{~mol})$ was added and refluxed for $3 \mathrm{~h}$. After cooling, the separated product was filtered and washed. Product was crystallized from ethanol (95\%).

2-(3'-(4-nitrophenyl)-1',5-diphenyl-3,4-dihydro-1'H,2H-[3,4'-bipyrazol]-2-yl)thiazol4(5H)-one (5a)

Yield: 64\%; m.p.: 263-265 ${ }^{\circ} \mathrm{C}$; IR (KBr, cm $\left.{ }^{-1}\right)$ : 3068, 3042 (C-H, aromatic), 2860 (C-H, methylene), $1690(\mathrm{C}=\mathrm{O}), 1578(\mathrm{C}=\mathrm{N}), 1532(\mathrm{C}=\mathrm{C}), 1485,1351\left(\mathrm{~N}-\mathrm{O}\right.$ asymmetric, symmetric, $\left.-\mathrm{NO}_{2}\right) ;{ }^{1} \mathrm{H}$ NMR (400 MHz, DMSO- $\left.d_{6}, \delta, \mathrm{ppm}\right): 3.42\left(\mathrm{dd}, 1 \mathrm{H}, J=17.50 \mathrm{~Hz}, 3.11 \mathrm{~Hz}, \mathrm{C}_{4}-\mathrm{H}\right.$ pyrazoline), 3.91 (dd, $1 \mathrm{H}, J=17.52 \mathrm{~Hz}, 11.10 \mathrm{~Hz}, \mathrm{C}_{4}-\mathrm{H}$ pyrazoline $), 4.11\left(\mathrm{~s}, 2 \mathrm{H}\right.$, thiazolone- $\left.\mathrm{C}_{5}-\mathrm{H}\right), 6.09(\mathrm{dd}, 1 \mathrm{H}, J=$ $11.15 \mathrm{~Hz}, 3.11 \mathrm{~Hz}, \mathrm{C}_{5}-\mathrm{H}$ pyrazoline), 6.86-8.21 (m, $\left.14 \mathrm{H}, \mathrm{Ar}-\mathrm{H}\right), 8.32\left(\mathrm{~s}, 1 \mathrm{H}\right.$, pyrazole-H); ${ }^{13} \mathrm{C}$ NMR (100 MHz, DMSO- $\left.d_{6}, \delta, \mathrm{ppm}\right): 39.0\left(\mathrm{C}_{5}\right.$ of thiazolone), $42.3\left(\mathrm{C}_{4}\right.$ of pyrazoline), $60.4\left(\mathrm{C}_{5}\right.$ of pyrazoline), 115.4-151.3 (Ar-C), $159.5(\mathrm{C}=\mathrm{N}$ of pyrazoline), $178.0(\mathrm{C}=\mathrm{N}$ of thiazolone $), 187.4$ $\left(\mathrm{C}=\mathrm{O}\right.$ of thiazolone); LCMS (m/z): $508.13\left(\mathrm{M}^{+}\right)$; Anal. Calcd. For $\mathrm{C}_{27} \mathrm{H}_{20} \mathrm{~N}_{6} \mathrm{O}_{3} \mathrm{~S}: \mathrm{C}-63.77, \mathrm{H}-3.96$, N-16.53; Found: C-63.59, H-3.84, N-16.44\%.

2-(5-(4-bromophenyl)-3'-(4-nitrophenyl)-1'-phenyl-3,4-dihydro-1'H,2H-[3,4'-bipyrazol]2-yl)thiazol-4(5H)-one (5b)

Yield: $62 \%$; m.p.: $191-193{ }^{\circ} \mathrm{C}$; IR $\left(\mathrm{KBr}, \mathrm{cm}^{-1}\right): 3065,3040$ (C-H, aromatic), 2866 (C-H, methylene), $1694(\mathrm{C}=\mathrm{O}), 1574(\mathrm{C}=\mathrm{N}), 1530(\mathrm{C}=\mathrm{C}), 1488,1354\left(\mathrm{~N}-\mathrm{O}\right.$ asymmetric, symmetric, $\left.-\mathrm{NO}_{2}\right), 664(-\mathrm{C}-$ $\mathrm{Br}) ;{ }^{1} \mathrm{H}$ NMR $\left(400 \mathrm{MHz}, \mathrm{DMSO}-d_{6}, \delta, \mathrm{ppm}\right): 3.39\left(\mathrm{dd}, 1 \mathrm{H}, J=17.52 \mathrm{~Hz}, 3.15 \mathrm{~Hz}, \mathrm{C}_{4}-\mathrm{H}\right.$ pyrazoline), $3.91\left(\mathrm{dd}, 1 \mathrm{H}, J=17.51 \mathrm{~Hz}, 11.11 \mathrm{~Hz}, \mathrm{C}_{4}-\mathrm{H}\right.$ pyrazoline $), 4.12\left(\mathrm{~s}, 2 \mathrm{H}\right.$, thiazolone- $\left.\mathrm{C}_{5}-\mathrm{H}\right)$, $6.09\left(\mathrm{dd}, 1 \mathrm{H}, J=11.13 \mathrm{~Hz}, 3.13 \mathrm{~Hz}, \mathrm{C}_{5}-\mathrm{H}\right.$ pyrazoline $), 6.92-8.17(\mathrm{~m}, 13 \mathrm{H}, \mathrm{Ar}-\mathrm{H}), 8.38(\mathrm{~s}, 1 \mathrm{H}$, pyrazole-H); ${ }^{13} \mathrm{C}$ NMR $\left(100 \mathrm{MHz}, \mathrm{DMSO}-d_{6}, \delta, \mathrm{ppm}\right): 39.4\left(\mathrm{C}_{5}\right.$ of thiazolone), $42.6\left(\mathrm{C}_{4}\right.$ of pyrazoline $), 60.4\left(\mathrm{C}_{5}\right.$ of pyrazoline $), 115.1-151.5(\mathrm{Ar}-\mathrm{C}), 159.5(\mathrm{C}=\mathrm{N}$ of pyrazoline $), 178.4(\mathrm{C}=\mathrm{N}$ of thiazolone), $187.1 \quad\left(\mathrm{C}=\mathrm{O}\right.$ of thiazolone); $\mathrm{LCMS}(\mathrm{m} / \mathrm{z}): 586.04\left(\mathrm{M}^{+}\right)$; Anal. Calcd. For $\mathrm{C}_{27} \mathrm{H}_{19} \mathrm{BrN}_{6} \mathrm{O}_{3} \mathrm{~S}$ : C-55.20, H-3.26, N-14.31; Found: C-55.36, H-3.40, N-14.22\%.

\section{2-(5-(2-bromophenyl)-3'-(4-nitrophenyl)-1'-phenyl-3,4-dihydro-1'H,2H-[3,4'-bipyrazol]-}

\section{2-yl)thiazol-4(5H)-one (5c)}

Yield: 58\%; m.p.: $175-177^{\circ} \mathrm{C}$; IR (KBr, cm $\left.{ }^{-1}\right): 3068,3039$ (C-H, aromatic), 2869 (C-H, methylene), $1690(\mathrm{C}=\mathrm{O}), 1571(\mathrm{C}=\mathrm{N}), 1535(\mathrm{C}=\mathrm{C}), 1480,1351\left(\mathrm{~N}-\mathrm{O}\right.$ asymmetric, symmetric, $\left.-\mathrm{NO}_{2}\right), 669(-\mathrm{C}-$ $\mathrm{Br}) ;{ }^{1} \mathrm{H}$ NMR $\left(400 \mathrm{MHz}, \mathrm{DMSO}-d_{6}, \delta, \mathrm{ppm}\right): 3.35\left(\mathrm{dd}, 1 \mathrm{H}, J=17.48 \mathrm{~Hz}, 3.08 \mathrm{~Hz}, \mathrm{C}_{4}-\mathrm{H}\right.$ pyrazoline), $3.94\left(\mathrm{dd}, 1 \mathrm{H}, J=17.50 \mathrm{~Hz}, 11.07 \mathrm{~Hz}, \mathrm{C}_{4}-\mathrm{H}\right.$ pyrazoline), 4.10 (s, 2H, thiazolone- $\left.\mathrm{C}_{5}-\mathrm{H}\right)$, $6.01\left(\mathrm{dd}, 1 \mathrm{H}, J=11.11 \mathrm{~Hz}, 3.17 \mathrm{~Hz}, \mathrm{C}_{5}-\mathrm{H}\right.$ pyrazoline), 6.98-8.20 (m, 13H, Ar-H), $8.41(\mathrm{~s}, 1 \mathrm{H}$, pyrazole-H); ${ }^{13} \mathrm{C}$ NMR $\left(100 \mathrm{MHz}, \mathrm{DMSO}-d_{6}, \delta, \mathrm{ppm}\right): 39.7\left(\mathrm{C}_{5}\right.$ of thiazolone $), 42.9\left(\mathrm{C}_{4}\right.$ of pyrazoline), 60.2 ( $\mathrm{C}_{5}$ of pyrazoline), 114.7-151.4 (Ar-C), 159.1 ( $\mathrm{C}=\mathrm{N}$ of pyrazoline $), 178.0(\mathrm{C}=\mathrm{N}$ of 
thiazolone), $187.4 \quad\left(\mathrm{C}=\mathrm{O}\right.$ of thiazolone); LCMS (m/z): $586.04\left(\mathrm{M}^{+}\right)$; Anal. Calcd. For $\mathrm{C}_{27} \mathrm{H}_{19} \mathrm{BrN}_{6} \mathrm{O}_{3} \mathrm{~S}$ : C-55.20, H-3.26, N-14.31; Found: C-55.48, H-3.37, N-14.46\%.

2-(5-(4-fluorophenyl)-3'-(4-nitrophenyl)-1'-phenyl-3,4-dihydro-1'H,2H-[3,4'-bipyrazol]2-yl)thiazol-4(5H)-one (5d)

Yield: 66\%; m.p.: 200-202 ${ }^{\circ} \mathrm{C}$; IR ( $\left.\mathrm{KBr}, \mathrm{cm}^{-1}\right)$ : 3066, 3037 (C-H, aromatic), 2862 (C-H, methylene), $1695(\mathrm{C}=\mathrm{O}), 1570(\mathrm{C}=\mathrm{N}), 1531(\mathrm{C}=\mathrm{C}), 1493,1354\left(\mathrm{~N}-\mathrm{O}\right.$ asymmetric, symmetric, $\left.-\mathrm{NO}_{2}\right), 1118(\mathrm{C}-$ F); ${ }^{1} \mathrm{H}$ NMR (400 MHz, DMSO- $\left.d_{6}, \delta, \mathrm{ppm}\right): 3.40$ (dd, $1 \mathrm{H}, J=17.49 \mathrm{~Hz}, 3.05 \mathrm{~Hz}, \mathrm{C}_{4}-\mathrm{H}$ pyrazoline), $3.94\left(\mathrm{dd}, 1 \mathrm{H}, J=17.48 \mathrm{~Hz}, 11.07 \mathrm{~Hz}, \mathrm{C}_{4}-\mathrm{H}\right.$ pyrazoline), $4.15\left(\mathrm{~s}, 2 \mathrm{H}\right.$, thiazolone- $\left.\mathrm{C}_{5}-\mathrm{H}\right), 6.00(\mathrm{dd}$, $1 \mathrm{H}, J=11.21 \mathrm{~Hz}, 3.09 \mathrm{~Hz}, \mathrm{C}_{5}-\mathrm{H}$ pyrazoline), 7.01-8.24 (m, 13H, Ar-H), 8.40 (s, 1H, pyrazole-H); ${ }^{13} \mathrm{C}$ NMR (100 MHz, DMSO- $\left.d_{6}, \delta, \mathrm{ppm}\right): 39.1\left(\mathrm{C}_{5}\right.$ of thiazolone), 42.2 ( $\mathrm{C}_{4}$ of pyrazoline), $60.3\left(\mathrm{C}_{5}\right.$ of pyrazoline), 114.5-151.5 (Ar-C), $159.0(\mathrm{C}=\mathrm{N}$ of pyrazoline $), 178.7(\mathrm{C}=\mathrm{N}$ of thiazolone $), 187.4$ $\left(\mathrm{C}=\mathrm{O}\right.$ of thiazolone); LCMS (m/z): $526.12\left(\mathrm{M}^{+}\right)$; Anal. Calcd. For $\mathrm{C}_{27} \mathrm{H}_{19} \mathrm{FN}_{6} \mathrm{O}_{3} \mathrm{~S}: \mathrm{C}-61.59, \mathrm{H}-3.64$, N-15.96; Found: C-61.64, H-3.71, N-15.84\%.

\section{2-(5-(3,5-difluorophenyl)-3'-(4-nitrophenyl)-1'-phenyl-3,4-dihydro-1'H,2H-[3,4'-bipyrazol]- 2-yl)thiazol-4(5H)-one (5e)}

Yield: $67 \%$; m.p.: $252-254{ }^{\circ} \mathrm{C}$; IR $\left(\mathrm{KBr}, \mathrm{cm}^{-1}\right)$ : 3067, 3041 (C-H, aromatic), 2869 (C-H, methylene), $1705(\mathrm{C}=\mathrm{O}), 1577(\mathrm{C}=\mathrm{N}), 1535(\mathrm{C}=\mathrm{C}), 1494,1350\left(\mathrm{~N}-\mathrm{O}\right.$ asymmetric, symmetric, $\left.-\mathrm{NO}_{2}\right), 1125(\mathrm{C}-$ F); ${ }^{1} \mathrm{H}$ NMR (400 MHz, DMSO- $\left.d_{6}, \delta, \mathrm{ppm}\right): 3.49$ (dd, $1 \mathrm{H}, J=17.48 \mathrm{~Hz}, 3.11 \mathrm{~Hz}, \mathrm{C}_{4}-\mathrm{H}$ pyrazoline), $3.97\left(\mathrm{dd}, 1 \mathrm{H}, J=17.40 \mathrm{~Hz}, 11.01 \mathrm{~Hz}, \mathrm{C}_{4}-\mathrm{H}\right.$ pyrazoline), 4.18 (s, 2H, thiazolone- $\left.\mathrm{C}_{5}-\mathrm{H}\right), 6.08$ (dd, $1 \mathrm{H}, J=11.20 \mathrm{~Hz}, 3.17 \mathrm{~Hz}, \mathrm{C}_{5}-\mathrm{H}$ pyrazoline), 7.10-8.20 (m, 12H, Ar-H), 8.41 (s, 1H, pyrazole-H); ${ }^{13} \mathrm{C}$ NMR (100 MHz, DMSO- $\left.d_{6}, \delta, \mathrm{ppm}\right): 39.0\left(\mathrm{C}_{5}\right.$ of thiazolone), $42.0\left(\mathrm{C}_{4}\right.$ of pyrazoline $), 61.3\left(\mathrm{C}_{5}\right.$ of pyrazoline), 114.7-152.7 (Ar-C), $158.1(\mathrm{C}=\mathrm{N}$ of pyrazoline $), 178.6(\mathrm{C}=\mathrm{N}$ of thiazolone $), 187.5$ $\left(\mathrm{C}=\mathrm{O}\right.$ of thiazolone); LCMS (m/z): $544.11\left(\mathrm{M}^{+}\right)$; Anal. Calcd. For $\mathrm{C}_{27} \mathrm{H}_{18} \mathrm{~F}_{2} \mathrm{~N}_{6} \mathrm{O}_{3} \mathrm{~S}$ : C-59.55, H3.33, N-15.43; Found: C-59.62, H-3.24, N-15.36\%.

\section{2-(5-(4-chlorophenyl)-3'-(4-nitrophenyl)-1'-phenyl-3,4-dihydro-1'H,2H-[3,4'-bipyrazol]- 2-yl)thiazol-4(5H)-one (5f)}

Yield: 75\%; m.p.: 274-276 ${ }^{\circ} \mathrm{C}$; IR (KBr, cm $\left.{ }^{-1}\right)$ : 3067, 3041 (C-H, aromatic), 2869 (C-H, methylene), $1705(\mathrm{C}=\mathrm{O}), 1577(\mathrm{C}=\mathrm{N}), 1535(\mathrm{C}=\mathrm{C}), 1494,1350\left(\mathrm{~N}-\mathrm{O}\right.$ asymmetric, symmetric, $\left.-\mathrm{NO}_{2}\right), 748(\mathrm{C}-$ $\mathrm{Cl}) ;{ }^{1} \mathrm{H}$ NMR $\left(400 \mathrm{MHz}, \mathrm{DMSO}-d_{6}, \delta, \mathrm{ppm}\right): 3.44\left(\mathrm{dd}, 1 \mathrm{H}, J=17.44 \mathrm{~Hz}, 3.14 \mathrm{~Hz}, \mathrm{C}_{4}-\mathrm{H}\right.$ pyrazoline), $3.94\left(\mathrm{dd}, 1 \mathrm{H}, J=17.45 \mathrm{~Hz}, 11.04 \mathrm{~Hz}, \mathrm{C}_{4}-\mathrm{H}\right.$ pyrazoline $), 4.15\left(\mathrm{~s}, 2 \mathrm{H}\right.$, thiazolone- $\left.\mathrm{C}_{5}-\mathrm{H}\right)$, $6.07\left(\mathrm{dd}, 1 \mathrm{H}, J=11.14 \mathrm{~Hz}, 3.15 \mathrm{~Hz}, \mathrm{C}_{5}-\mathrm{H}\right.$ pyrazoline $), 7.00-8.22(\mathrm{~m}, 13 \mathrm{H}, \mathrm{Ar}-\mathrm{H}), 8.35(\mathrm{~s}, 1 \mathrm{H}$, pyrazole-H); ${ }^{13} \mathrm{C}$ NMR $\left(100 \mathrm{MHz}, \mathrm{DMSO}-d_{6}, \delta, \mathrm{ppm}\right): 39.5\left(\mathrm{C}_{5}\right.$ of thiazolone), $42.8\left(\mathrm{C}_{4}\right.$ of pyrazoline $), 61.1\left(\mathrm{C}_{5}\right.$ of pyrazoline $), 114.5-151.8(\mathrm{Ar}-\mathrm{C}), 158.7(\mathrm{C}=\mathrm{N}$ of pyrazoline $), 178.4(\mathrm{C}=\mathrm{N}$ of thiazolone), $187.0 \quad\left(\mathrm{C}=\mathrm{O}\right.$ of thiazolone); LCMS $(\mathrm{m} / \mathrm{z}): 542.09\left(\mathrm{M}^{+}\right) ;$Anal. Calcd. For $\mathrm{C}_{27} \mathrm{H}_{19} \mathrm{ClN}_{6} \mathrm{O}_{3} \mathrm{~S}$ : C-59.72, H-3.53, N-15.48; Found: C-59.63, H-3.45, N-15.34\%.

\section{2-(5-(2-chlorophenyl)-3'-(4-nitrophenyl)-1'-phenyl-3,4-dihydro-1'H,2H-[3,4'-bipyrazol]-} 2-yl)thiazol-4(5H)-one (5g)

Yield: 70\%; m.p.: 207-209 ${ }^{\circ} \mathrm{C}$; IR (KBr, cm $\left.{ }^{-1}\right)$ : 3069, 3040 (C-H, aromatic), 2867 (C-H, methylene), $1709(\mathrm{C}=\mathrm{O}), 1570(\mathrm{C}=\mathrm{N}), 1534(\mathrm{C}=\mathrm{C}), 1497,1351\left(\mathrm{~N}-\mathrm{O}\right.$ asymmetric, symmetric, $\left.-\mathrm{NO}_{2}\right), 740(\mathrm{C}-$ $\mathrm{Cl}) ;{ }^{1} \mathrm{H}$ NMR $\left(400 \mathrm{MHz}, \mathrm{DMSO}-d_{6}, \delta, \mathrm{ppm}\right): 3.40\left(\mathrm{dd}, 1 \mathrm{H}, J=17.40 \mathrm{~Hz}, 3.10 \mathrm{~Hz}, \mathrm{C}_{4}-\mathrm{H}\right.$ pyrazoline), $3.90\left(\mathrm{dd}, 1 \mathrm{H}, J=17.41 \mathrm{~Hz}, 11.07 \mathrm{~Hz}, \mathrm{C}_{4}-\mathrm{H}\right.$ pyrazoline), $4.13\left(\mathrm{~s}, 2 \mathrm{H}\right.$, thiazolone- $\left.\mathrm{C}_{5}-\mathrm{H}\right)$, $6.05\left(\mathrm{dd}, 1 \mathrm{H}, J=11.14 \mathrm{~Hz}, 3.15 \mathrm{~Hz}, \mathrm{C}_{5}-\mathrm{H}\right.$ pyrazoline $), 7.01-8.24(\mathrm{~m}, 13 \mathrm{H}, \mathrm{Ar}-\mathrm{H}), 8.37(\mathrm{~s}, 1 \mathrm{H}$, pyrazole-H); ${ }^{13} \mathrm{C}$ NMR $\left(100 \mathrm{MHz}, \mathrm{DMSO}-d_{6}, \delta, \mathrm{ppm}\right): 39.8\left(\mathrm{C}_{5}\right.$ of thiazolone), $42.4\left(\mathrm{C}_{4}\right.$ of pyrazoline $), 61.2\left(\mathrm{C}_{5}\right.$ of pyrazoline $), 115.2-151.7(\mathrm{Ar}-\mathrm{C}), 158.1(\mathrm{C}=\mathrm{N}$ of pyrazoline $), 178.7(\mathrm{C}=\mathrm{N}$ of thiazolone $), \quad 187.4\left(\mathrm{C}=\mathrm{O}\right.$ of thiazolone); LCMS $(\mathrm{m} / \mathrm{z}): 542.09\left(\mathrm{M}^{+}\right)$; Anal. Calcd. For $\mathrm{C}_{27} \mathrm{H}_{19} \mathrm{ClN}_{6} \mathrm{O}_{3} \mathrm{~S}$ : C-59.72, H-3.53, N-15.48; Found: C-59.65, H-3.48, N-15.39\%.

2-(5-(2,4-dichlorophenyl)-3'-(4-nitrophenyl)-1'-phenyl-3,4-dihydro-1'H,2H-[3,4'-bipyrazol]-2yl)thiazol-4(5H)-one (5h)

Yield: 69\%; m.p.: $240-242{ }^{\circ} \mathrm{C}$; IR (KBr, cm $\left.{ }^{-1}\right): 3074,3044$ (C-H, aromatic), 2869 (C-H, methylene), $1700(\mathrm{C}=\mathrm{O}), 1577(\mathrm{C}=\mathrm{N}), 1536(\mathrm{C}=\mathrm{C}), 1498,1353\left(\mathrm{~N}-\mathrm{O}\right.$ asymmetric, symmetric, $\left.-\mathrm{NO}_{2}\right), 754(\mathrm{C}-$ 
$\mathrm{Cl}) ;{ }^{1} \mathrm{H}$ NMR (400 MHz, DMSO- $\left.d_{6}, \delta, \mathrm{ppm}\right): 3.48\left(\mathrm{dd}, 1 \mathrm{H}, J=17.45 \mathrm{~Hz}, 3.04 \mathrm{~Hz}, \mathrm{C}_{4}-\mathrm{H}\right.$ pyrazoline), $3.86\left(\mathrm{dd}, 1 \mathrm{H}, J=17.42 \mathrm{~Hz}, 11.10 \mathrm{~Hz}, \mathrm{C}_{4}-\mathrm{H}\right.$ pyrazoline $), 4.10\left(\mathrm{~s}, 2 \mathrm{H}\right.$, thiazolone- $\left.\mathrm{C}_{5}-\mathrm{H}\right)$, $6.01\left(\mathrm{dd}, 1 \mathrm{H}, J=11.16 \mathrm{~Hz}, 3.17 \mathrm{~Hz}, \mathrm{C}_{5}-\mathrm{H}\right.$ pyrazoline), 7.03-8.28 (m, 12H, Ar-H), $8.34(\mathrm{~s}, 1 \mathrm{H}$, pyrazole-H); ${ }^{13} \mathrm{C}$ NMR $\left(100 \mathrm{MHz}, \mathrm{DMSO}-d_{6}, \delta, \mathrm{ppm}\right): 39.5\left(\mathrm{C}_{5}\right.$ of thiazolone), $42.7\left(\mathrm{C}_{4}\right.$ of pyrazoline), $61.4\left(\mathrm{C}_{5}\right.$ of pyrazoline), 115.0-151.1 (Ar-C), $158.4(\mathrm{C}=\mathrm{N}$ of pyrazoline $), 178.5(\mathrm{C}=\mathrm{N}$ of thiazolone), $187.2\left(\mathrm{C}=\mathrm{O}\right.$ of thiazolone); LCMS $(\mathrm{m} / \mathrm{z}): 576.05\left(\mathrm{M}^{+}\right)$; Anal. Calcd. For $\mathrm{C}_{27} \mathrm{H}_{18} \mathrm{Cl}_{2} \mathrm{~N}_{6} \mathrm{O}_{3} \mathrm{~S}$ : C-56.16, H-3.14, N-14.55; Found: C-56.28, H-3.26, N-14.68\%.

\section{2-(5-(3,4-dichlorophenyl)-3'-(4-nitrophenyl)-1'-phenyl-3,4-dihydro-1'H,2H-[3,4'-bipyrazol]-2- yl)thiazol-4(5H)-one (5i)}

Yield: 73\%; m.p.: $144-146{ }^{\circ} \mathrm{C}$; IR ( $\left.\mathrm{KBr}, \mathrm{cm}^{-1}\right)$ : 3077, 3046 (C-H, aromatic), 2872 (C-H, methylene), $1704(\mathrm{C}=\mathrm{O}), 1579(\mathrm{C}=\mathrm{N}), 1537(\mathrm{C}=\mathrm{C}), 1487,1350\left(\mathrm{~N}-\mathrm{O}\right.$ asymmetric, symmetric, $\left.-\mathrm{NO}_{2}\right), 759(\mathrm{C}-$ $\mathrm{Cl}) ;{ }^{1} \mathrm{H}$ NMR $\left(400 \mathrm{MHz}, \mathrm{DMSO}-d_{6}, \delta, \mathrm{ppm}\right): 3.44\left(\mathrm{dd}, 1 \mathrm{H}, J=17.40 \mathrm{~Hz}, 3.05 \mathrm{~Hz}, \mathrm{C}_{4}-\mathrm{H}\right.$ pyrazoline), $3.87\left(\mathrm{dd}, 1 \mathrm{H}, J=17.40 \mathrm{~Hz}, 11.08 \mathrm{~Hz}, \mathrm{C}_{4}-\mathrm{H}\right.$ pyrazoline), $4.14\left(\mathrm{~s}, 2 \mathrm{H}\right.$, thiazolone-C $\left.\mathrm{C}_{5}-\mathrm{H}\right)$, $6.04\left(\mathrm{dd}, 1 \mathrm{H}, J=11.15 \mathrm{~Hz}, 3.15 \mathrm{~Hz}, \mathrm{C}_{5}-\mathrm{H}\right.$ pyrazoline), 7.01-8.20 (m, 12H, Ar-H), $8.36(\mathrm{~s}, 1 \mathrm{H}$, pyrazole-H); ${ }^{13} \mathrm{C}$ NMR $\left(100 \mathrm{MHz}, \mathrm{DMSO}-d_{6}, \delta, \mathrm{ppm}\right): 39.7\left(\mathrm{C}_{5}\right.$ of thiazolone), $42.5\left(\mathrm{C}_{4}\right.$ of pyrazoline $), 61.7\left(\mathrm{C}_{5}\right.$ of pyrazoline $), 115.1-152.3(\mathrm{Ar}-\mathrm{C}), 158.5(\mathrm{C}=\mathrm{N}$ of pyrazoline $), 178.0(\mathrm{C}=\mathrm{N}$ of thiazolone), $187.1 \quad\left(\mathrm{C}=\mathrm{O}\right.$ of thiazolone); $\mathrm{LCMS}(\mathrm{m} / \mathrm{z}): 576.05\left(\mathrm{M}^{+}\right)$; Anal. Calcd. For $\mathrm{C}_{27} \mathrm{H}_{18} \mathrm{Cl}_{2} \mathrm{~N}_{6} \mathrm{O}_{3} \mathrm{~S}$ : C-56.16, H-3.14, N-14.55; Found: C-56.30, H-3.22, N-14.43\%.

\section{2-(3'-(4-nitrophenyl)-1'-phenyl-5-(p-tolyl)-3,4-dihydro-1'H,2H-[3,4'-bipyrazol]-2-yl)thiazol- $4(5 \mathrm{H})$-one (5j)}

Yield: 67\%; m.p.: $225-227^{\circ} \mathrm{C}$; IR (KBr, cm $\left.{ }^{-1}\right): 3077,3046\left(\mathrm{C}-\mathrm{H}\right.$, aromatic), $2930\left(\mathrm{C}-\mathrm{H}, \mathrm{CH}_{3}\right), 2872$ (C-H, methylene), $1704(\mathrm{C}=\mathrm{O}), 1579(\mathrm{C}=\mathrm{N}), 1537(\mathrm{C}=\mathrm{C}), 1487,1350$ (N-O asymmetric, symmetric, $\left.-\mathrm{NO}_{2}\right) ;{ }^{1} \mathrm{H}$ NMR $\left(400 \mathrm{MHz}, \mathrm{DMSO}-d_{6}, \delta, \mathrm{ppm}\right): 2.46\left(\mathrm{~s}, 3 \mathrm{H}, \mathrm{CH}_{3}\right), 3.41(\mathrm{dd}, 1 \mathrm{H}, J=$ $17.41 \mathrm{~Hz}, 3.01 \mathrm{~Hz}, \mathrm{C}_{4}-\mathrm{H}$ pyrazoline), $3.85\left(\mathrm{dd}, 1 \mathrm{H}, J=17.44 \mathrm{~Hz}, 11.02 \mathrm{~Hz}, \mathrm{C}_{4}-\mathrm{H}\right.$ pyrazoline), 4.11 (s, 2H, thiazolone- $\left.\mathrm{C}_{5}-\mathrm{H}\right), 6.03\left(\mathrm{dd}, 1 \mathrm{H}, J=11.15 \mathrm{~Hz}, 3.14 \mathrm{~Hz}, \mathrm{C}_{5}-\mathrm{H}\right.$ pyrazoline), 7.12-8.27 (m, $13 \mathrm{H}, \mathrm{Ar}-\mathrm{H}), 8.42$ (s, $1 \mathrm{H}$, pyrazole-H); ${ }^{13} \mathrm{C}$ NMR (100 MHz, DMSO-d $6, \delta$, ppm): $21.4\left(-\mathrm{CH}_{3}\right), 39.5$ $\left(\mathrm{C}_{5}\right.$ of thiazolone), 42.4 ( $\mathrm{C}_{4}$ of pyrazoline), $61.6\left(\mathrm{C}_{5}\right.$ of pyrazoline), 115.6-152.9 (Ar-C), 158.6 $(\mathrm{C}=\mathrm{N}$ of pyrazoline), $178.7(\mathrm{C}=\mathrm{N}$ of thiazolone $), 187.5(\mathrm{C}=\mathrm{O}$ of thiazolone $) ; \mathrm{LCMS}(\mathrm{m} / \mathrm{z}): 522.15$ $\left(\mathrm{M}^{+}\right)$; Anal. Calcd. For $\mathrm{C}_{28} \mathrm{H}_{22} \mathrm{~N}_{6} \mathrm{O}_{3} \mathrm{~S}$ : C-64.35, H-4.24, N-16.08; Found: C-64.32, H-4.19, N$16.15 \%$.

\section{2-(3',5-bis(4-nitrophenyl)-1'-phenyl-3,4-dihydro-1'H,2H-[3,4'-bipyrazol]-2-yl)thiazol-4(5H)-one}

(5k)

Yield: 56\%; m.p.: 235-237 ${ }^{\circ} \mathrm{C}$; IR (KBr, cm $\left.{ }^{-1}\right): 3072,3045$ (C-H, aromatic), 2870 (C-H, methylene), $1695(\mathrm{C}=\mathrm{O}), 1574(\mathrm{C}=\mathrm{N}), 1535(\mathrm{C}=\mathrm{C}), 1490,1351\left(\mathrm{~N}-\mathrm{O}\right.$ asymmetric, symmetric, $\left.-\mathrm{NO}_{2}\right) ;{ }^{1} \mathrm{H}$ NMR (400 MHz, DMSO- $\left.d_{6}, \delta, \mathrm{ppm}\right): 3.40\left(\mathrm{dd}, 1 \mathrm{H}, J=17.42 \mathrm{~Hz}, 3.07 \mathrm{~Hz}, \mathrm{C}_{4}-\mathrm{H}\right.$ pyrazoline), 3.81 (dd, $1 \mathrm{H}, J=17.42 \mathrm{~Hz}, 11.02 \mathrm{~Hz}, \mathrm{C}_{4}-\mathrm{H}$ pyrazoline $), 4.10(\mathrm{~s}, 2 \mathrm{H}$, thiazolone-C $5-\mathrm{H}), 6.00(\mathrm{dd}, 1 \mathrm{H}, J=$ $11.13 \mathrm{~Hz}, 3.12 \mathrm{~Hz}, \mathrm{C}_{5}-\mathrm{H}$ pyrazoline), 7.14-8.30 (m, $\left.13 \mathrm{H}, \mathrm{Ar}-\mathrm{H}\right), 8.41\left(\mathrm{~s}, 1 \mathrm{H}\right.$, pyrazole-H); ${ }^{13} \mathrm{C}$ NMR (100 MHz, DMSO- $\left.d_{6}, \delta, \mathrm{ppm}\right): 39.0\left(\mathrm{C}_{5}\right.$ of thiazolone), $41.9\left(\mathrm{C}_{4}\right.$ of pyrazoline), $61.4\left(\mathrm{C}_{5}\right.$ of pyrazoline), 114.9-153.1 (Ar-C), $158.2(\mathrm{C}=\mathrm{N}$ of pyrazoline), $178.4(\mathrm{C}=\mathrm{N}$ of thiazolone), 187.5 $\left(\mathrm{C}=\mathrm{O}\right.$ of thiazolone); LCMS (m/z): $553.12\left(\mathrm{M}^{+}\right)$; Anal. Calcd. For $\mathrm{C}_{27} \mathrm{H}_{19} \mathrm{~N}_{7} \mathrm{O}_{5} \mathrm{~S}: \mathrm{C}-58.58, \mathrm{H}-3.46$, N-17.71; Found: C-58.65, H-3.35, N-17.86\%.

\section{2-(5-(3-nitrophenyl)-3'-(4-nitrophenyl)-1'-phenyl-3,4-dihydro-1'H,2H-[3,4'-bipyrazol]-}

\section{2-yl)thiazol-4(5H)-one (5l)}

Yield: 61\%; m.p.: $259-261{ }^{\circ} \mathrm{C}$; IR (KBr, cm $\left.{ }^{-1}\right)$ : 3075, 3042 (C-H, aromatic), 2872 (C-H, methylene), $1696(\mathrm{C}=\mathrm{O}), 1571(\mathrm{C}=\mathrm{N}), 1533(\mathrm{C}=\mathrm{C}), 1493,1352\left(\mathrm{~N}-\mathrm{O}\right.$ asymmetric, symmetric, $\left.-\mathrm{NO}_{2}\right)$; ${ }^{1} \mathrm{H}$ NMR (400 MHz, DMSO- $d_{6}, \delta, \mathrm{ppm}$ ): $3.43\left(\mathrm{dd}, 1 \mathrm{H}, J=17.40 \mathrm{~Hz}, 3.02 \mathrm{~Hz}, \mathrm{C}_{4}-\mathrm{H}\right.$ pyrazoline), 3.82 (dd, $1 \mathrm{H}, J=17.43 \mathrm{~Hz}, 11.01 \mathrm{~Hz}, \mathrm{C}_{4}-\mathrm{H}$ pyrazoline), $4.12\left(\mathrm{~s}, 2 \mathrm{H}\right.$, thiazolone-C $\left.\mathrm{C}_{5}-\mathrm{H}\right), 5.99(\mathrm{dd}, 1 \mathrm{H}, J=$ $11.15 \mathrm{~Hz}, 3.10 \mathrm{~Hz}, \mathrm{C}_{5}-\mathrm{H}$ pyrazoline), 7.12-8.35 (m, 13H, Ar-H), $8.40\left(\mathrm{~s}, 1 \mathrm{H}\right.$, pyrazole-H); ${ }^{13} \mathrm{C}$ NMR (100 MHz, DMSO- $\left.d_{6}, \delta, \mathrm{ppm}\right): 39.6\left(\mathrm{C}_{5}\right.$ of thiazolone), $42.0\left(\mathrm{C}_{4}\right.$ of pyrazoline), $61.7\left(\mathrm{C}_{5}\right.$ of pyrazoline), 114.1-153.2 (Ar-C), $157.8(\mathrm{C}=\mathrm{N}$ of pyrazoline), $178.3(\mathrm{C}=\mathrm{N}$ of thiazolone), 187.2 
$\left(\mathrm{C}=\mathrm{O}\right.$ of thiazolone); LCMS (m/z): $553.12\left(\mathrm{M}^{+}\right)$; Anal. Calcd. For $\mathrm{C}_{27} \mathrm{H}_{19} \mathrm{~N}_{7} \mathrm{O}_{5} \mathrm{~S}$ : C-58.58, H-3.46, N-17.71; Found: C-58.47, H-3.56, N-17.65\%.

\section{2-(5-(4-methoxyphenyl)-3'-(4-nitrophenyl)-1'-phenyl-3,4-dihydro-1'H,2H-[3,4'-bipyrazol]- 2-yl)thiazol-4(5H)-one (5m)}

Yield: $67 \%$; m.p.: $225-227{ }^{\circ} \mathrm{C}$; IR ( $\left.\mathrm{KBr}, \mathrm{cm}^{-1}\right)$ : 3071, 3050 (C-H, aromatic), 2870 (C-H, methylene), $2840\left(-\mathrm{OCH}_{3}\right), 1710(\mathrm{C}=\mathrm{O}), 1571(\mathrm{C}=\mathrm{N}), 1533(\mathrm{C}=\mathrm{C}), 1488,1352(\mathrm{~N}-\mathrm{O}$ asymmetric, symmetric, $\left.\mathrm{NO}_{2}\right) ;{ }^{1} \mathrm{H}$ NMR $\left(400 \mathrm{MHz}, \mathrm{DMSO}-d_{6}, \delta, \mathrm{ppm}\right): 3.38\left(\mathrm{dd}, 1 \mathrm{H}, J=17.40 \mathrm{~Hz}, 3.00 \mathrm{~Hz}, \mathrm{C}_{4}-\mathrm{H}\right.$ pyrazoline), $3.82\left(\mathrm{dd}, 1 \mathrm{H}, J=17.44 \mathrm{~Hz}, 11.00 \mathrm{~Hz}, \mathrm{C}_{4}-\mathrm{H}\right.$ pyrazoline), $3.88\left(\mathrm{~s}, 3 \mathrm{H}, \mathrm{OCH}_{3}\right), 4.12(\mathrm{~s}$, $2 \mathrm{H}$, thiazolone- $\left.\mathrm{C}_{5}-\mathrm{H}\right), 6.02\left(\mathrm{dd}, 1 \mathrm{H}, J=11.10 \mathrm{~Hz}, 3.14 \mathrm{~Hz}, \mathrm{C}_{5}-\mathrm{H}\right.$ pyrazoline), 7.00-8.20 (m, 13H, Ar-H), 8.43 (s, $1 \mathrm{H}$, pyrazole-H); ${ }^{13} \mathrm{C}$ NMR (100 MHz, DMSO- $\left.d_{6}, \delta, \mathrm{ppm}\right): 39.1\left(\mathrm{C}_{5}\right.$ of thiazolone), $42.0\left(\mathrm{C}_{4}\right.$ of pyrazoline), $55.7\left(-\mathrm{OCH}_{3}\right), 61.2\left(\mathrm{C}_{5}\right.$ of pyrazoline $), 115.1-153.2(\mathrm{Ar}-\mathrm{C}), 158.7(\mathrm{C}=\mathrm{N}$ of pyrazoline), $178.9\left(\mathrm{C}=\mathrm{N}\right.$ of thiazolone), $187.1\left(\mathrm{C}=\mathrm{O}\right.$ of thiazolone); LCMS (m/z): $538.14\left(\mathrm{M}^{+}\right)$; Anal. Calcd. For $\mathrm{C}_{28} \mathrm{H}_{22} \mathrm{~N}_{6} \mathrm{O}_{4} \mathrm{~S}$ : C-62.44, H-4.12, N-15.60; Found: C-62.35, H-4.23, N-15.65\%.

\section{2-(5-(3-methoxyphenyl)-3'-(4-nitrophenyl)-1'-phenyl-3,4-dihydro-1'H,2H-[3,4'-bipyrazol]- 2-yl)thiazol-4(5H)-one (5n)}

Yield: 68\%; m.p.: $229-231{ }^{\circ} \mathrm{C}$; IR ( $\left.\mathrm{KBr}, \mathrm{cm}^{-1}\right)$ : 3074, 3052 (C-H, aromatic), 2877 (C-H, methylene), $2844\left(-\mathrm{OCH}_{3}\right), 1711(\mathrm{C}=\mathrm{O}), 1572(\mathrm{C}=\mathrm{N}), 1534(\mathrm{C}=\mathrm{C}), 1485,1355(\mathrm{~N}-\mathrm{O}$ asymmetric, symmetric, $\left.\mathrm{NO}_{2}\right) ;{ }^{1} \mathrm{H}$ NMR (400 MHz, DMSO- $\left.d_{6}, \delta, \mathrm{ppm}\right): 3.37\left(\mathrm{dd}, 1 \mathrm{H}, J=17.42 \mathrm{~Hz}, 3.04 \mathrm{~Hz}, \mathrm{C}_{4}-\mathrm{H}\right.$ pyrazoline), $3.80\left(\mathrm{dd}, 1 \mathrm{H}, J=17.41 \mathrm{~Hz}, 11.02 \mathrm{~Hz}, \mathrm{C}_{4}-\mathrm{H}\right.$ pyrazoline), $3.86\left(\mathrm{~s}, 3 \mathrm{H}, \mathrm{OCH}_{3}\right), 4.11(\mathrm{~s}$, $2 \mathrm{H}$, thiazolone- $\left.\mathrm{C}_{5}-\mathrm{H}\right), 6.05\left(\mathrm{dd}, 1 \mathrm{H}, J=11.12 \mathrm{~Hz}, 3.11 \mathrm{~Hz}, \mathrm{C}_{5}-\mathrm{H}\right.$ pyrazoline), 7.03-8.27 (m, 13H, Ar-H), 8.45 (s, $1 \mathrm{H}$, pyrazole-H); ${ }^{13} \mathrm{C}$ NMR (100 MHz, DMSO- $\left.d_{6}, \delta, \mathrm{ppm}\right): 39.3$ ( $\mathrm{C}_{5}$ of thiazolone), $42.4\left(\mathrm{C}_{4}\right.$ of pyrazoline), $55.0\left(-\mathrm{OCH}_{3}\right), 61.5\left(\mathrm{C}_{5}\right.$ of pyrazoline $), 114.8-153.4(\mathrm{Ar}-\mathrm{C}), 158.4(\mathrm{C}=\mathrm{N}$ of pyrazoline), $178.4\left(\mathrm{C}=\mathrm{N}\right.$ of thiazolone), $187.0\left(\mathrm{C}=\mathrm{O}\right.$ of thiazolone); LCMS (m/z): $538.14\left(\mathrm{M}^{+}\right)$; Anal. Calcd. For $\mathrm{C}_{28} \mathrm{H}_{22} \mathrm{~N}_{6} \mathrm{O}_{4} \mathrm{~S}$ : C-62.44, H-4.12, N-15.60; Found: C-62.40, H-4.26, N-15.63\%.

\section{2-(5-(2-methoxyphenyl)-3'-(4-nitrophenyl)-1'-phenyl-3,4-dihydro-1'H,2H-[3,4'-bipyrazol]- 2-yl)thiazol-4(5H)-one (5o)}

Yield: 63\%; m.p.: 208-210 ${ }^{\circ} \mathrm{C}$; IR (KBr, $\left.\mathrm{cm}^{-1}\right)$ : 3075, 3055 (C-H, aromatic), 2875 (C-H, methylene), $2840\left(-\mathrm{OCH}_{3}\right), 1712(\mathrm{C}=\mathrm{O}), 1571(\mathrm{C}=\mathrm{N}), 1533(\mathrm{C}=\mathrm{C}), 1481,1357(\mathrm{~N}-\mathrm{O}$ asymmetric, symmetric, $\left.\mathrm{NO}_{2}\right) ;{ }^{1} \mathrm{H}$ NMR (400 MHz, DMSO- $\left.d_{6}, \delta, \mathrm{ppm}\right): 3.34\left(\mathrm{dd}, 1 \mathrm{H}, J=17.47 \mathrm{~Hz}, 3.08 \mathrm{~Hz}, \mathrm{C}_{4}-\mathrm{H}\right.$ pyrazoline), $3.81\left(\mathrm{dd}, 1 \mathrm{H}, J=17.42 \mathrm{~Hz}, 11.07 \mathrm{~Hz}, \mathrm{C}_{4}-\mathrm{H}\right.$ pyrazoline), $3.88\left(\mathrm{~s}, 3 \mathrm{H}, \mathrm{OCH}_{3}\right), 4.12(\mathrm{~s}$, $2 \mathrm{H}$, thiazolone- $\left.\mathrm{C}_{5}-\mathrm{H}\right), 6.03\left(\mathrm{dd}, 1 \mathrm{H}, J=11.10 \mathrm{~Hz}, 3.13 \mathrm{~Hz}, \mathrm{C}_{5}-\mathrm{H}\right.$ pyrazoline $), 7.10-8.26(\mathrm{~m}, 13 \mathrm{H}$, Ar-H), 8.42 (s, $1 \mathrm{H}$, pyrazole-H); ${ }^{13} \mathrm{C}$ NMR (100 MHz, DMSO- $\left.d_{6}, \delta, \mathrm{ppm}\right): 39.6\left(\mathrm{C}_{5}\right.$ of thiazolone), $42.8\left(\mathrm{C}_{4}\right.$ of pyrazoline), $55.5\left(-\mathrm{OCH}_{3}\right), 61.7\left(\mathrm{C}_{5}\right.$ of pyrazoline $), 114.7-153.5(\mathrm{Ar}-\mathrm{C}), 158.1(\mathrm{C}=\mathrm{N}$ of pyrazoline), $178.2\left(\mathrm{C}=\mathrm{N}\right.$ of thiazolone), $187.2\left(\mathrm{C}=\mathrm{O}\right.$ of thiazolone); LCMS (m/z): $538.14\left(\mathrm{M}^{+}\right)$; Anal. Calcd. For $\mathrm{C}_{28} \mathrm{H}_{22} \mathrm{~N}_{6} \mathrm{O}_{4} \mathrm{~S}$ : C-62.44, H-4.12, N-15.60; Found: C-62.34, H-4.12, N-15.54\%.

\section{Conclusion}

The comparative study of these catalytic synthesis had shown that $\mathrm{Cs}_{2} \mathrm{CO}_{3}$ is the best suitable heterogeneous catalyst for the synthesis of 2-(3'-(4-nitrophenyl)-1'-phenyl-5-(aryl)-3,4-dihydro1' $H, 2 H$-[3,4'-bipyrazol]-2-yl)thiazol-4(5H)-ones moieties. Synthesized compounds had shown significant activity. It may be visualized from the activity results that halogen derivatives are identified as the most potential agents against bacterial strains and fungal strains.

\section{Acknowledgements}

Authors are thankful to the University Grants Commission, New Delhi and Department of Science \& Technology, New Delhi for financial support under the NON-SAP and DST-FIST programs respectively. 


\section{References}

[1] E. Akbas, I. Berber, Antibacterial and antifungal activities of new pyrazolo[3,4-d]pyridazin derivatives, Eur. J. Med. Chem. 40 (2005) 401-405.

[2] S. Manfredini, R. Bazzanini, P.G. Baraldi, M. Guarneri, D. Simoni, M.E. Marongiu, A. Pani, P.L. Colla, E. Tramontano, Pyrazole-related nucleosides. Synthesis and antiviral/antitumor activity of some substituted pyrazole and pyrazolo[4,3-d]-1,2,3-triazin-4-one nucleosides, J. Med. Chem. 35 (1992) 917-924.

[3] C.W. Noell, C.C. Cheng, Pyrazoles. 4. Analogs of 3-(3,3-dimethyl-1-triazeno)pyrazole-4carboxamide, J. Med. Chem. 14 (1971) 1245-1246.

[4] M. Abdel-Aziz, G.A. Abuo-Rahma, A.A. Hassan, Synthesis of novel pyrazole derivatives and evaluation of their antidepressant and anticonvulsant activities, Eur. J. Med. Chem.44 (2009) 34803487.

[5] Z. Ozdemir, H.B. Kandilici, B. Gumusel, U. Calis, A. Bilgin, Synthesis and studies on antidepressant and anticonvulsant activities of some 3-(2-furyl)-pyrazoline derivatives, Eur. J. Med. Chem.42 (2007) 373-379.

[6] S.Y. Hassan, Synthesis, antibacterial and antifungal activity of some new pyrazoline and pyrazole derivatives, Molecules. 3 (2013) 2683-2711.

[7] R.B. Pathak, P.T. Chovatia, H.H. Parekh, Synthesis, antitubercular and antimicrobial evaluation of 3-(4-chlorophenyl)-4-substituted pyrazole derivatives, Bioorg Med Chem Lett. 22 (2012) 51295133.

[8] A. Palomer, F. Cabre, J. Pascual, J. Campos, M.A. Trujillo, A. Entrena, M.A. Gallo, L. Garcia, D. Mauleon, A. Espinosa, dentification of novel cyclooxygenase-2 selective inhibitors using pharmacophore models, J. Med. Chem. 45 (2002) 1402-1411.

[9] D.J. Wustrow, T. Capiris, R. Rubin, J.A. Knobelsdorf, H. Akunne, M.D. Davis, R. MacKenzie, T.A. Pugsley, K.T. Zoski, T.G. Heffner, L.D. Wise, Pyrazolo[1,5-a]pyrimidine CRF-1 receptor antagonists, Bioorg. Med. Chem. Lett. 18 (1998) 2067-2070.

[10] T.D. Penning, J. J Talley, S.R. Bertenshaw, J.S. Carter, P.W. Collins, S.Docter, M.J. Graneto, L.F. Lee, J.W. Malecha, J.M. Miyashiro, R.S. Rogers, D.J. Rogier, S.S. Yu, G.D. Anderson, E.G. Burton, J.N. Cogburn, S.A. Gregory, C.M. Koboldt, W.E. Perkins, K.Seibert, A.W. Veenhuizen, Y.Y. Zhang, P.C. Isakson, Pyrazolo[1,5-a]pyrimidine CRF-1 receptor antagonists, J. Med. Chem. 40 (1997), 1347-1365.

[11] S.R. Joshi, P.G. Mandhane, S.D. Diwakar, S.K. Dabhade, C.H. Gill, Synthesis, analgesic and anti-inflammatory activities of some novel pyrazolines derivatives, Bioorg. Med. Chem. Lett. 20 (2010) 3721-3725.

[12] A.R. Bhat, F. Athar, A. Azam, New derivatives of 3,5-substituted-1,4,2-dioxazoles: synthesis and activity against Entamoeba histolytica, Eur. J. Med. Chem. 44 (2009) 926-936.

[13] Z. Ozdemir, H.B. KandilcI, B. Gümüşel, U. Caliş, A.A. Bilgin, Synthesis and studies on antidepressant and anticonvulsant activities of some 3-(2-furyl)-pyrazoline derivatives, Eur J Med Chem. 42 (2007) 373-379.

[14] K. Manna, Y.K. Agrawal, Microwave assisted synthesis of new indophenazine 1,3,5trisubstruted pyrazoline derivatives of benzofuran and their antimicrobial activity, Bioorg. Med. Chem. Lett. 19 (2009) 2688-2692.

[15] Z.A. Kaplancikli, G. Turan-Zitouni, A. Ozdemir, O.D. Can, P. Chevallet, Synthesis and antinociceptive activities of some pyrazoline derivatives, Eur. J. Med. Chem. 44 (2009) 2606-2610. [16] A. Marella, M. Shaquiquzzaman, M. Akhter, G. Verma, M.M. Alam, Novel pyrazolepyrazoline hybrids endowed with thioamide as antimalarial agents: their synthesis and 3D-QSAR studies , J Enzyme Inhib Med Chem, 30 (2015) 597-606.

[17] I. Braulio, T. Alexis, O. Fabian, Q. Jairo, A. Rodrigo, N. Manuel, S. Adolfo, C. Justo, Synthesis of novel pyrazolic analogues of chalcones and their 3-aryl-4-(3-aryl-4,5-dihydro-1Hpyrazol-5-yl)-1-phenyl-1H-pyrazole derivatives as potential antitumor agents, Bioorg. Med. Chem. 18 (2010) 4965-4974. 
[18] T.S. Jeong, K.S. Kim, J.R. Kim, K.Y. Cho, S. Lee, W.S. Lee, Novel 3,5-diaryl pyrazolines and pyrazole as low-density lipoprotein (LDL) oxidation inhibitor, Bioorg. Med. Chem. Lett. 14 (2004) 2719-2723.

[19] A. Budakoti, A.R. Bhat, A. Azam, Synthesis of new 2-(5-substituted-3-phenyl-2-pyrazolinyl)1,3-thiazolino[5,4-b]quinoxaline derivatives and evaluation of their antiamoebic activity, Eur. J. Med. Chem. 44 (2009) 1317-1325.

[20] H. Faisal, S. Attar, U. Sadiq, A. Amir, Synthesis, characterization, antiamoebic activity and cytotoxicity of novel 2-(quinolin-8-yloxy) acetohydrazones and their cyclized products (1,2,3thiadiazole and 1,2,3-selenadiazole derivatives), Eur. J. Med. Chem. 45 (2010) 6127-6134.

[21] Z. Ratkovic, Z.D. Juranic, T. Stanojkovic, D. Manojlovic, R.D. Vukicevic, N. Radulovic, M.D. Joksović, Synthesis, characterization, electrochemical studies and antitumor activity of some new chalcone analogues containing ferrocenyl pyrazole moiety, Bioorg. Chem. 38 (2010) 26-32.

[22] A.A.Bekhit, T. Abdel-Aziem, Design, synthesis and biological evaluation of some pyrazole derivatives as anti-inflammatory-antimicrobial agents, Bioorg. Med. Chem. 12 (2004) 1935-1945.

[23] A. Kaplancikli, G. Turan-Zitouni, A. Ozdemir, Synthesis and antinociceptive activities of some pyrazoline derivatives, Eur. J. Med. Chem. 44 (2009) 2606-2610.

[24] M.G. Mamolo, D. Zampieri, V. Falagiani, L.Vio, E. Banfi. Synthesis and antimycobacterial activity of 5-aryl-1-isonicotinoyl-3-(pyridin-2-yl)-4, 5-dihydro-1H-pyrazole derivatives, Farmaco 56 (2001) 593-599.

[25] H. Khalilullah, S. Khan, M.J. Ahsan, B. Ahmed, Synthesis and antihepatotoxic activity of 5(2,3-dihydro-1,4-benzodioxane-6-yl)-3-substituted-phenyl-4,5-dihydro-1H-pyrazole derivatives, Bioorg. Med. Chem. Lett, 21 (2011) 7251-7254.

[26] M.A. Berghot, E.B. Moawad, Convergent synthesis and antibacterial activity of pyrazole and pyrazoline derivatives of diazepam, Eur. J. Pharm. Sci. 20 (2003) 173-179.

[27] L. Gomez, M.D. Hack, J. Wu J.J.M. Wiener, H. Venkatesan, Jr. A. Santillan, D.J. Pippel, N.Mani, B.J. Morrow, S.T. Motley, K.J. Shaw, R.Wolin, C.A.Grice, T.K. Jones, Novel pyrazole derivatives as potent inhibitors of type II topoisomerases. Part 1: synthesis and preliminary SAR analysis, Bioorg. Med. Chem. Lett. 17 (2007) 2723-2727.

[28] A.Tanitame,Y. Oyamada, K. Ofuji, M. Fujimoto, N. Iwai, Y. Hiyama, K Suzuki, H. Ito, K. Nagai, M. Wachi, J.I. Yamagishi, Synthesis and antibacterial activity of a novel series of potent DNA gyrase inhibitors. Pyrazole derivatives, J. Med. Chem. 47 (2004) 3693-3696.

[29] B.J. Bradbury, M.J. Pucci, Recent advances in bacterial topoisomerase inhibitors, Curr. Opin. Pharmacol. 8 (2008) 574-581.

[30] N. Siddiqui, S.K. Arya, W. Ahsan, B. Azad, Diverse biological activities of Thiazoles: A Retrospect, Int. j. drug dev. Res, 3 (2001) 55-67.

[31] R. Lesyk, B. S. Zimenkovsky, 4-Thiazolidones: centenarian history, current status and perspectives for modern organic and medicinal chemistry, Curr. Org. Chem. 8 (2004) 1547-1577.

[32] N. C. Desai, N. Shihory, M. Bhatt, B. Patel, T. Karkar, Studies on Antimicrobial Evaluation of Some 1-((1-(1H-Benzo[d]imidazol-2-yl)ethylidene)amino)-6-((arylidene)amino)-2-oxo-4-phenyl-1, 2-dihydropyridine-3,5-dicarbonitriles, Synth Commun, 45 (2015) 27012711.

[33] N. C. Desai, N. Bhatt, A. Dodiya, T. Karkar, B. Patel, M. Bhatt, Synthesis, characterization and antimicrobial screening of thiazole based 1,3,4-oxadiazoles heterocycles, Res. Chem. Intermed. (2016) 3039 -3053.

[34] N. C. Desai V. V. Joshi, K. M. Rajpara, H. V. Vaghani, H.M. Satodiya, Facile synthesis of novel fluorine containing pyrazole based thiazole derivatives and evaluation of antimicrobial activity, J. Fluorine Chem. 142 (2012) 67-78. 